\title{
TRAFFIC SIGNAL CONTROL WITH VEHICLE-TO-EVERYTHING COMMUNICATION
}

\author{
Muntaser A. Salman ${ }^{1,2}$,Suat Ozdemir ${ }^{3}$ and Fatih V. Celebi ${ }^{2}$ \\ ${ }^{1}$ Department of Information Systems, University of Anbar, Anbar, Iraq \\ ${ }^{2}$ Computer Engineering Department,YildirimBeyazit University,Ankara,Turkey \\ ${ }^{3}$ Computer Engineering Department, Gazi University, Ankara, Turkey
}

\begin{abstract}
Traffic signal control (TSC) with vehicle-to-everything(V2X) communication can be very efficient for solving traffic congestion problem. When regarding a low number of vehicles equipped with communication capability i.e. penetration rate (PR), an assumption that TSC can operate with a sufficient quality need to be studied. In this paper, this assumption was investigated with simulations using COLOMBO framework. The PR is the major factor that influences the quality of TSC, but as well the evaluation interval should be taken into account. The performance of TSC in means of sufficient period should follow the evaluations of the overall system. COLOMBO framework has been further investigated and new two approaches have been proposed (i.e. instead of swarm algorithm used in COLOMBO framework, simple and fuzzy logic have been used). To evaluate the performance of our proposal, a comparison with COLOMBO's approaches have been done. The results suggested that the duration that a vehicle remains associated with roadside unit (RSU) directly or through group leader can be used for controlling (as well as evaluating the traffic conditions) of an intersection with good accuracy even for low PR.
\end{abstract}

\section{KEYWORDS}

Traffic Signal Control, Vehicle to Everything Communication, Penetration Rate, COLOMBO Framework, Swarm algorithms \& Fuzzy Logic.

\section{INTRODUCTION}

Traffic signals are able to self-organize and adapt to traffic conditions changing through vehicular communications monitoring and intelligent control algorithm. Vehicular communication monitoring provide extensive information of approaching vehicles as they frequently transmit a specific messages (e.g. basic safety message (BSM) in United States (US), or cooperative awareness message (CAM) in Europe countries(EU)), containing all required relevant information (e.g. speed, position, ...etc.). A convenient method to acquire data from cooperative vehicles is to receive messages with RSU. After receiving the message, the RSU extract the most important information that is suitable for TSC. Then, controlling an intersection through TSC required an algorithm that can deal with the RSU's information sufficiently.

In US and EU, several researches have been conducted for TSC of an intersection using vehicular communication protocols. Generally speaking, based on PR, two different approach for TSC can

Natarajan Meghanathan et al. (Eds) : NeTCoM, CSEIT, GRAPH-HOC, NCS, SIPR - 2017

pp. 57-69, 2017. (C) CS \& IT-CSCP 2017

DOI : $10.5121 /$ csit.2017.71506 
be observed. Either full penetration rate(FPR) or partial penetration rate (PPR). This is done based on how much vehicular communication data are sufficient in the computation that achieve (full or partial) knowledge of the traffic conditions.

FPR-based approach is built upon an assumption that all vehicles are capable of communicate with each other for compressing the description about the traffic conditions. With FPR approach, it is capable to determine traffic conditions indicators without involving a RSU. Thereby, it may be applied for virtual TSC (or virtual traffic light VTL) of an intersection without real traffic signals infrastructure. In this context, VTL project [1] explored the benefits of vehicle-to-vehicle (V2V) communication for VTL using elected leader. For which, the responsibility of creating the VTL and broadcasting the traffic signal messages is assigned. The main drawback for this approach is the FPR that assumed. Although VTL project contributors in [2] show that VTLs can offer benefits in both throughput and delay with partial deployment scenarios, but they suggest an external representation for the VTL. Yet aspects such as visibility, and legislation, were not addressed. Clearly, these issues will play a major role in deciding what is the most appropriate representation and as a result a deployment issue is grown.

On the contrary, PPR-based approach is built upon an assumption that some vehicles are capable of communicate with each other and the available traffic signals infrastructure involve RSU. With PPR approach, to overcome the partial knowledge of traffic conditions, TSC can become more benefit with intelligent algorithm. In this context, the idea of self-organizing algorithm is merged in 2005 by Gershenson [3]. It was demonstrated that traffic signals are able to self-organize and adapt to changing traffic conditions by using simple rules without direct communication among intersections. The simple self-organizing traffic lights algorithm proposed in [3] gives preference to vehicles that have been waiting longer, and to larger groups of vehicles (platoons). According to that approach, platoons affect the behaviour of traffic signals operation, prompting them to turn green.

The idea of exploiting vehicular communication is merging into the field of self-organizing TSC recently. For instance, in[4] a decentralized adaptive TSC algorithm using V2I communication data was developed. Their algorithm was phase based and the objective was to minimize total queue length. The control problem considered as an optimization problem and it was solved by dynamic programming. In [5] a platoon based TSC algorithm in a vehicular communication environment was proposed. The algorithm divided a phase into two stages where the first stage served standing queue and the second stage served vehicles approaching the intersection. Based on the location and speed, the travel time of the vehicles can be obtained. Result showed that $40 \%$ PR was critical for effectiveness of the algorithm. In [6] a TSC framework for multi-modal under V2I communication was proposed. Their algorithm was platoon based and the objective was formulated to solve for an optimal signal plan based on current traffic condition, controller status, platoon data and priority requests. In [7] acumulative travel-time responsive real time intersection control algorithm with vehicular communication data was presented. The algorithm applied a kalman filter to estimate cumulative travel time under low PR. The phasing with highest combined travel time was set to be the next phase. The paper stated that at least $30 \%$ PR was required. In [8] a predictive microscopic simulation algorithm for TSC was proposed. The algorithm took data from vehicles including positions, headings and speeds and imported them to a model to predict the future traffic conditions. A rolling horizon strategy was chosen to optimize either delay only or a combination of delay, stops and deceleration. The algorithm considered several PRs as well as estimating the states of unequipped vehicles based on equipped vehicle states. Previous researches [3]-[8] showed that the PR was a critical parameter in determining the effectiveness of the TSC algorithms.

From literature review, it is clear that there are inefficiencies and trade-offs under different PR (e.g. efficient use of the communications channel versus accurate TSC and traffic conditions 
estimation) that need to be focus on. In this context, the EU FP7 COLOMBO (2012-2015) project [9] exploits V2X protocols in the context of TSC. This was done in order to determine traffic surveillance information about local queue length in proximity of traffic signals. The goal of which is to use such traffic indicators to dynamically adapt TSC algorithms and timings. COLOMBO focuses on TSC algorithms using swarm algorithm with V2X cooperative data.

In this paper, simple and fuzzy logic had been investigated in COLOMBO framework instead of swarm algorithm with more detailed information that cooperative V2X protocol can offer.

The remainder of this paper is organized as follows. Section 2, presents COLOMBO project efforts in the field of intelligent TSC using V2X protocol with low PR. Section 3, describes in details the perspective of the approach proposed in this paper. Section 3 analyses the approach performance and reports the primary experimental results collected so far. Comparison with related works presented in Section 4. Discussions with on ongoing research and conclusive remarks end the paper.

\section{COLOMBO's TSC}

The main effort of the COLOMBO project is dedicated towards making self-organizing traffic lights an effective means for practical TSC [9]. The common underlying principle of the policies developed for the COLOMBO project is the teach TSC, controlling one or more interconnected intersections, operates independently of all other controllers and gets information only on the traffic flow on its incoming and outgoing lanes. Ideally, a birds-eye view of the traffic network with speed, position and route information about each vehicle should be available. Using this information, the TSC knows exactly how many vehicles are waiting or approaching each signal group. Both traditional detection V2X protocols and standard cooperative systems cannot deliver this even with FPR-based approach. This means that TSC algorithms rely on estimates of traffic conditions to divide the arrival flow between signal groups. With PPR-based approach, one could coarsely assume that absolute numbers (like number of vehicle and sum of stops) can be hardly determined, while averaging measures (like average speed) can be retrieved with a sufficient quality. In this context, COLOMBO project [9], developed an open source framework and confirm the previous assumption using simulations. COLOMBO framework use swarm intelligence algorithm to estimate an abstract of traffic conditions (called it pheromone) based on average speed and its derivative. Based on these pheromones, different TSC methods (i.e policies such as phase, platoon, marching and congestion policy) are developed. Each policy performs under specific traffic condition and not for others. For example, phase policy terminates the current phase as soon as another one has reached the traffic threshold after the minimum duration constraint of the current stage is satisfied. This policy was designed to handle medium-low traffic conditions, where this early termination would not make the TSC switch too often. It is worth mentioning that phase policy never ends the current stage if there are no cars opposing the currently allowed traffic flow.

Platoon policy tries to let all the vehicles in the currently green lanes pass the intersection before releasing the green light. It is worth noting that even the platoon policy will not switch phase unless another one requests the green light. The maximum phase duration is taken into account in order to pre-empt the current phase execution even if there are approaching vehicles.

In intense traffic conditions, each phase will be executed for the maximum allowed time. The definition of the maximum allowed time for a phase greatly impacts the performance of the system. 
Marching policy is adequate when no vehicle is sensed or when the traffic looks too intense from all directions to take any online decision regarding the input lanes. In this case, there are two possible approaches:

- Falling back to a static duration for each stages;

- Taking into account the traffic conditions of the outgoing lanes to prevent traffic towards already heavily loaded ones.

The approach chosen by COLOMBO framework developer is the first one because they want the policies to implement simple rules.

Finally, congestion policy is used when the output lanes are congested and there may be vehicles waiting in front of the intersection. To avoid gridlocks, all input lanes are inhibited, i.e. the system terminates the current phase executing each stage for their minimum duration time. When the all red light stage is reached, no other phase is activated until the congestion has been solved.

To this aim, the goal of the policy selection procedure is to select which policy should be executed in the TSC undercurrent traffic conditions. In order to do that, COLOMBO framework has a large number of parameters that need to be appropriately set to achieve best possible performance. A parameter tuning optimization approach is required. It is time consuming and required a lot of traffic data to be aggregated in static or dynamic approach to overcome the whole traffic conditions. One drawback of this approach comes from the local traffic conditions sample aggregation areas. In static approaches the challenge is to determine the zone length that is neither too large nor too small. Dynamic approaches adjust to true traffic conditions, the challenge is to build and maintain dynamic clusters and cluster leaders.

On the other hand, in COLOMBO framework, a set of vehicles that are approaching an intersection are grouped following a common direction as an intermediate level of abstraction between the RSU and the multitude of surrounding vehicles. From this grouping; the group leader is chosen in an approximate central position, so that it can reach all other peers by simple singlehop communication and coordinate all group members. This grouping and group leader approximation chosen procedures in V2X protocols of COLOMBO framework make the dynamic cluster, with different PRs and traffic conditions, fuzzy and uncertain. Instead of using swarm algorithm to abstract traffic conditions, fuzzy logic had been used in this paper to estimate accumulative delay time with respect to the total travel time. Such indicators can be directly used for estimating the traffic conditions. This estimation, based on average speed and its derivative, is investigated with different PR for intelligent TSC design.

\section{PROPOSED TSC}

In this paper, we propose to follow PPR-based with different strategy. We can do that by estimating accumulative delay with respect to their total travel time as a direct indicator to the traffic conditions estimation. Our approach uses the V2X protocol that used in COLOMBO framework, mainly for average speed estimation purposes, to estimate the accumulative delay time for each edge per moment (e.g. in our simulation per second) using fuzzy logic. These conditions are monitored and accumulated locally by the RSU of an intersection. When a different edge situation is detected, the average of the individual estimations is determined in order to collaboratively detect and characterize the whole intersection traffic conditions. The relation between the averages of estimated accumulative delay time can be used directly to make a TSC adaptive. The details of our TSC algorithm is going to be explained in details in the following subsections. 


\subsection{Traffic conditions estimation}

In PPR-based approach, PR is neither known nor can be estimated for near future. Because of that, the estimated average speed of vehicles per edge and its derivative can be use to estimate the accumulative delay time for each approaching edge, as well as for the whole edges, of an intersection per moment. Estimating the accumulative delay time (with respect to total travel time) is not just for traffic conditions estimation but also give an indicator for evaluation period for the intersection as a whole.

In general, delay time can be defined as the sum of acceleration, deceleration and stopped delay time. Where acceleration time can be defined as the time that determined with low speed and acceleration for vehicle (or group of vehicles) entered to the edge under the RSU coverage area. While, deceleration time can be defined as the time that determined with high speed and deceleration for vehicle (or group of vehicles)entered to the edge under the RSU coverage area. And finally, stopped delay time can be defined as the time that determined with zero speed and zero acceleration/deceleration for vehicle(or group of vehicles) entered to the edge under the RSU coverage area. This will be done in the RSU instantaneously per second so that the delay time per second can be determined.

Cumulative delay time can be used as a good indicator for evaluating TSC continually. For each second the information of the group is sent to the RSU that joins the intersection. The RSU use the incoming information for estimating the cumulative delay time of each edge separately. By averaging the cumulative delay time to the whole incoming edges of the intersection continuously, traffic conditions for the whole intersection can be estimated. As the vehicle travels along an intersection encounters different degrees of delay (i.e. different traffic conditions), so the value of the average cumulative delay time varies accordingly. Intuitively, the higher value of the average cumulative arriving time indicates the worse degree of traffic condition. Each RSU implementing our solution to estimates cumulative delay time based on its average vehicles speed and their derivative (acceleration/deceleration). The average vehicles speed can be easily obtained from the[1] protocol. Therefore, in each road (i.e. direction) we can estimate its cumulative delay time in terms of its average vehicles speed and their derivative through fuzzy system.

As in any fuzzy system, the input variables are first classified into different categories or fuzzy sets. The possible fuzzy sets for the speed are L for low, $\mathrm{M}$ for medium, and $\mathrm{H}$ for high. For the derivative, the defined fuzzy sets are $\mathrm{N}$ for negative, $\mathrm{Z}$ for zero, and $\mathrm{P}$ for positive. In addition, output fuzzy sets corresponding to estimated cumulative delay time have also been defined for one second time span, with $\mathrm{L}$ for low, $\mathrm{M}$ for medium and $\mathrm{H}$ for high. One of the main particularities of fuzzy logic is that a fuzzy set can contain elements with partial degree of membership, and consequently, an input value can belong to several fuzzy sets at the same time. For instance, a speed value of $9.9445 \mathrm{~m} / \mathrm{s}$ (i.e. with maximum edge speed equal to $13.889 \mathrm{~m} / \mathrm{s}$ ) could be member, with a different degree of membership, of both medium and high speed fuzzy sets.

In order to determine the degree of membership of the input values to each of the fuzzy sets, membership functions are employed. The membership functions used in our solution, which have been implemented based on simple rating system, are illustrated in Fig. 1(a), Fig. 1 (b) (with average acceleration $\mathrm{a}=2.8$ and deceleration $\mathrm{d}=4.8$ [10]) and Fig. 1(c).

To finalize the definition of our fuzzy system, fuzzy rules that relate the input (speed and its derivative) and the output fuzzy sets (delay time per second) have been established and are displayed in Table I. The fuzzy rules have been designed based on the speed, its derivative and arriving/leaving time physical relationship. As Fig. 1(c) illustrates, the output of the fuzzy system is a continuous value within the interval $[0,1]$ indicating the delay time, per second. 
In this case, some policies have to be check and select the most suitable one based on the traffic conditions of the incoming edge in an adaptation way. The details of which is given in the following subsection.

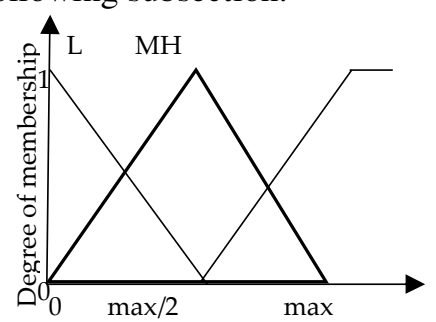

(a)

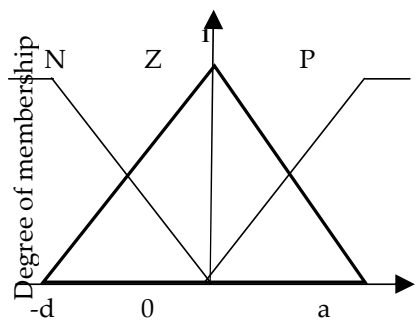

(b)

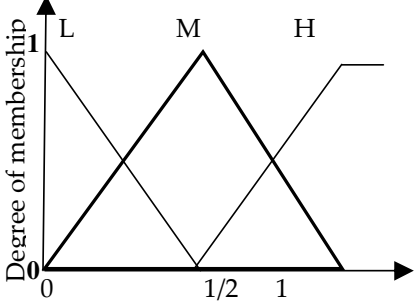

(c)

Figure 1. Fuzzy delay estimation system. (a) Average speed (m/s/veh) input sets. (b) Average speed derivative (m/s2/veh) input sets. (c) Average acceleration, deceleration and stopped delay (s/veh) outputs sets.

Table 1. Fuzzy rules relating inputs and outputs sets.

\begin{tabular}{|c|c|c|c|c|}
\hline \multicolumn{2}{|c|}{$\begin{array}{c}\text { Average acceleration/ deceleration } \\
\text { /stopped delay time (sec/veh) }\end{array}$} & \multicolumn{3}{|c|}{$\begin{array}{r}\text { Average speed derivative } \\
\left(\mathbf{m} / \mathbf{s}^{2} / \mathbf{v e h}\right)\end{array}$} \\
\cline { 3 - 5 } \multicolumn{2}{|c|}{} & $\mathbf{N}$ & $\mathbf{Z}$ & $\mathbf{P}$ \\
\hline $\begin{array}{c}\text { Average speed } \\
(\mathbf{m} / \mathbf{s} / \mathbf{v e h})\end{array}$ & $\mathbf{L}$ & $\mathrm{L} / \mathrm{H} / \mathrm{L}$ & $\mathrm{L} / \mathrm{L} / \mathrm{H}$ & $\mathrm{H} / \mathrm{L} / \mathrm{L}$ \\
\cline { 2 - 5 } & $\mathbf{H}$ & $\mathrm{L} / \mathrm{M} / \mathrm{L}$ & $\mathrm{L} / \mathrm{L} / \mathrm{M}$ & $\mathrm{M} / \mathrm{L} / \mathrm{L}$ \\
\cline { 2 - 5 } & $\mathrm{L} / \mathrm{L} / \mathrm{L}$ & $\mathrm{L} / \mathrm{L} / \mathrm{L}$ & $\mathrm{L} / \mathrm{L} / \mathrm{L}$ \\
\hline
\end{tabular}

\subsection{Traffic signal adaptation}

Self-organizing TSC is an online adaptation controller based on the individual estimations that different participating edges make locally through RSU. As described in the previous section, every RSU in an isolated intersection continuously monitors the individual traffic conditions for each approaching edge, and estimates through fuzzy system the current cumulative delay time. Only when the estimated of cumulative delay time exceeds a predefined threshold value, RSU activate the suitable control action (i.e. termination of the current state or not) as well as choosing the suitable policy (e.g. platoon, phase, marching and congestion policies used in COLOMBO project). Policies focus primarily on the duration of the current green stage. Each policy differs from the others mainly by the condition used to adjust the green time. Predefined threshold value may be corresponds to the level of service LOS of delay time to be monitored for each edge and/or for the intersection as a whole. At signalized intersections the motorized vehicles' LOS is a simple grading function of the average vehicle control delay. It may be calculated per intersection, per edge, or per lane group.

The adaptation mechanism is based on comparisons which are occurred when the leader updates the group fused data and sends it to the corresponding RSU. These comparisons are employed based on existing of vehicles and cumulative delay time made by different incoming edges. With predefined threshold value, traffic management policies can be changed in an adaptive way. In addition, the average cumulative delay time of last updating is exchanged to quantify the level of service for the intersection as a whole. Finally, RSU situated in the centre of an intersection will get a global and complete vision of the level of cumulative delay time for whole the approaching edges in the intersection. A key aspect in our solution is to identify the average cumulative delay time close to the RSU of the intersection that will change the adaptation procedure. Adaptation technique defines a procedure that is open for further optimization. The edge is considered to have increase value of cumulative delay time if its previous estimations sustainable reported some 
stayed vehicle from previous cycle, and such cycle is not operated well to clear all the vehicles. Every edge has a counter that represent accumulative delay time updated to the current moment according to the following equation:

$$
\begin{aligned}
D_{e}(k)= & \left\{\begin{array}{cc}
D_{e}(k-1)+\text { Delay }_{e}(k) & \text { if } \operatorname{car}_{e}(k)>0 \text { and } k \neq 0 \\
0 & \text { otherwise }
\end{array}\right. \\
& \text { with Delay }(k)=\sum_{i=1}^{3} F_{i}\left(v_{e}(k), \frac{d v_{e}(k)}{d k}\right)
\end{aligned}
$$

Where $D_{e}(k)$ and $D_{e}(k-1)$ are the new and the previous value of accumulative GTD for edge e at time-step $k$ and previous time-step $(k-1)$ respectively.Delay $(k)$ is the summation of estimated average acceleration $F_{1}$, deceleration $F_{2}$ and stopped delay $F_{3}$ respectively based on the fuzzy inference $F$ with edge average speed $v_{e}(k)$ and its derivative $d v_{e}(k) / d k$ as an inputs. Finally, $\operatorname{car}_{e}(k)$ is the number of vehicles in edge e at time-stepk.

Then, average of accumulative delay time for an intersection of $\mathrm{n}$ edges is given by:

$$
\begin{aligned}
& \operatorname{Avg} D_{e}(k)= \begin{cases}\operatorname{Avg} D_{\text {in }}(k) & \text { for incoming edge } \\
\operatorname{Avg} D_{\text {out }}(k) & \text { for outgoing edge }\end{cases} \\
& = \begin{cases}\frac{1}{n} \sum_{e=1}^{n} D_{e}(k) & \text { for } n \text { incoming edge } \\
\frac{1}{m} \sum_{e=1}^{m} D_{e}(k) & \text { for } m \text { outgoing edge }\end{cases}
\end{aligned}
$$

Since our solution has to be robust to low PRs, instead of using the counting number of cars, the updating of accumulative delay time is computed with only sensed car.

The policy selection algorithm makes use of previous indicator(i.e. $\left.D_{e}(k)\right)$ to decide which policy should be executed. Platoonand phase policies also use this measure to determine theduration of green period by applying a threshold (the so called traffic threshold) over it. Here threshold can be chosen as constant value (e.g. LOS required for the whole of the intersection or for each edge of it).

Based on [11], platoon and phase policies are not suitable for low traffic conditions. Swarm and platoon are the best policies for very high traffic conditions. Congestion policy is selected when all the inputs lanes are congested and there are no suitable decision based on available information or when the output lanes are congested and there are vehicles waiting in the intersection.

Thus, the following simple selection rules can be use:

- If $D_{e}(k)<10$ then Policy is "Marching"

- If $D_{e}(k) \geq 10$ and $D_{e}(k)<20$ then Policy is "Marching"

- If $D_{e}(k) \geq 20$ and $D_{e}(k)<35$ then Policy is "Marching"

- If $D_{e}(k) \geq 35$ and $D_{e}(k)<55$ then Policy is "Phase"

- If $D_{e}(k) \geq 55$ and $D_{e}(k)<80$ then Policy is "Platoon"

- If $D_{e}(k)>80$ then Policy is "Congestion"

One further issue should be mentioned here, delay time estimation is done instantaneously for each edge as well as for the whole intersection. The policy selection procedure of the TSC for the whole intersection should be rather insensitive to very short peaks delay time estimation, like a singular platoon in one edge, but should react rapidly to more persistent traffic changes where we expect a burst in traffic from a single direction that will last for specific period (e.g. fifteen to 
twenty minutes). In order to do that, total vehicles sensed time (VST)is proposed by the following equation:

$$
\operatorname{VST}_{e}(k)=\left\{\begin{array}{cc}
V S T_{e}(k)+1 & \text { if } \operatorname{car}_{e}(k)>0 \text { and } k \neq 0 \\
0 & \text { otherwise }
\end{array}\right.
$$

whereVST $(k)$ and $\operatorname{VST}_{e}(k-1)$ are the vehicle sensedtotal time per edge e at time $(k)$ and $(k-1)$ respectively. With this proposal, the percentage delay timecan be determined using the following equation:

$$
A D P_{e}(k) \%=D_{e}(k) / V S T_{e}(k)
$$

Where, $A D P_{e}(k) \%$ is the percentage delay time for edge $e$ attime $k$.

To this aim, every edge of an intersection evaluates its local delay time estimation. If the evaluation process continue for a certain period of time (typically 15 minutes)with acceptable (typically $A D P_{e}(k)<10 \%$ ), no changefor intersection TSC policy will required, otherwise policyselection procedure is activated to select new policy. The above description for policy selection procedure can be given by the following equation:

$$
\text { Terminate }=\left\{\begin{array}{rr}
Y e s & \text { if } \max _{e=1}^{n} A D P_{e}(k) \% \geq 0.1 \\
N o & \text { otherwise }
\end{array}\right.
$$

Where Treminate is the Boolean activation result for policy selection procedure. $\max _{e=1}^{n} A D P_{e}(k) \%$ is the maximum $A D P_{e}(k) \%$ for $n$ edge of an intersection. The results of thecurrent proposed approach are described in the followingsection.

\section{SimUlation RESULTS}

For evaluating the proposed TSC approach, a simple scenario consisting of an intersection was taken from COLOMBO framework (called Rilsa intersection [10], as shown in Fig. 2).This is done for two reason, comparability and traffic realistic.

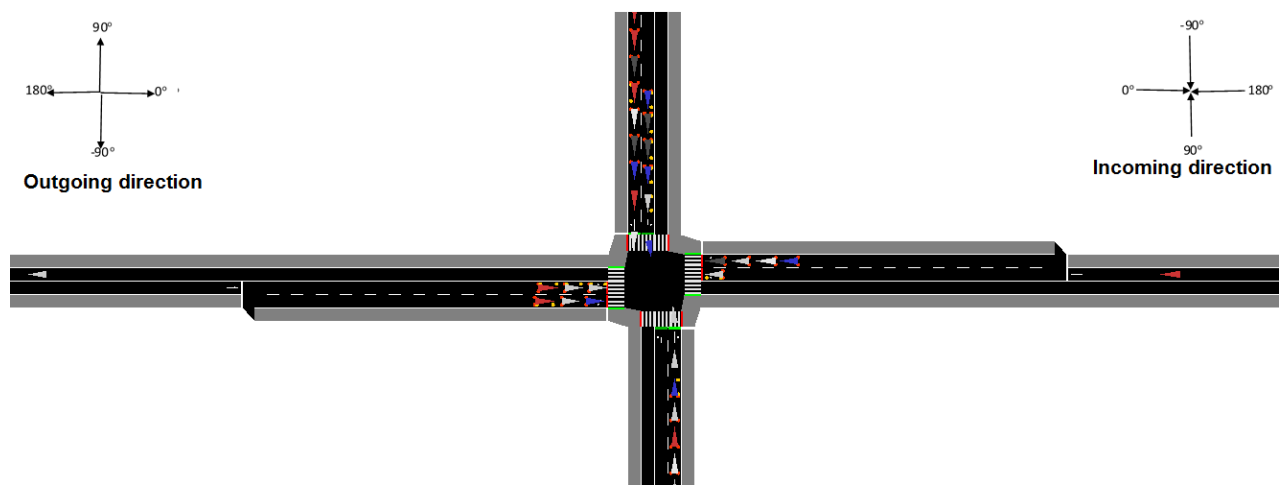

Figure 2. RILSA Intersection with incoming and outgoing direction

At the network level, all communications are performed by the ns-3 standard yans WiFi model using IEEE 802.11pwith ETSI ITS G5 standards. V2X communications with a fixed $170 \mathrm{~m}$ transmission range are assumed. The value of 170 meters is chosen to match the maximum communication range of a mobile node used in COLOMBO framework. A6 Mbps bandwidth rate with OFDM and default log-distance propagation model is used to compute signal loss.

In the simulation study, RSU periodically receive messages from group leader (if exist) within one second sampling resolution indicating number of cars and average speed per incoming and 
outgoing edge respectively. All simulations were performed in the same one hour time span. Vehicle densities are changed during time according to a wave trend that follows the green and red timings controlled by the traffic light. Table 3.reports the main configurations and parameters used in our simulations.

Table 3. Simulation parameters.

\begin{tabular}{|c|c|}
\hline Parameter & Value \\
\hline Wi-Fi mode & $802.11 \mathrm{p} /$ ETSI ITS 5G \\
\hline Transmission mode & $6 \mathrm{Mbps}(\mathrm{OFDM})$ \\
\hline Node radius & $170 \mathrm{~m}$ \\
\hline Propagation loss & Logarithmic \\
\hline Propagation speed & Constant $\left(3 \times 10^{8} \mathrm{~m} / \mathrm{s}\right)$ \\
\hline Penetration rate & $100,50,20,10,5,2,1 \%$ \\
\hline Simulation time & 1 hour \\
\hline
\end{tabular}

Vehicles densities and traffic conditions change during time according to a wave trend that follows the green and red timings controlled by the traffic signals. First, simulations have been run using one single policy at a time to compare with. The simulation involved the following policies: marching, platoon, phase and congestion. Then, COLOMBO framework with swarm algorithm as well as our approach (using fuzzy logic) are simulated. All of the above simulations had been run for measuring how the average waiting time, time loss and duration of the vehicles to accomplish their route varies, depending on the PR.

Average waiting time under different PR, shown in Fig. 3,depicts the number of steps in which the vehicle speed was below $0.1 \mathrm{~m} / \mathrm{s}$ measured in simulation steps from SUMO output.

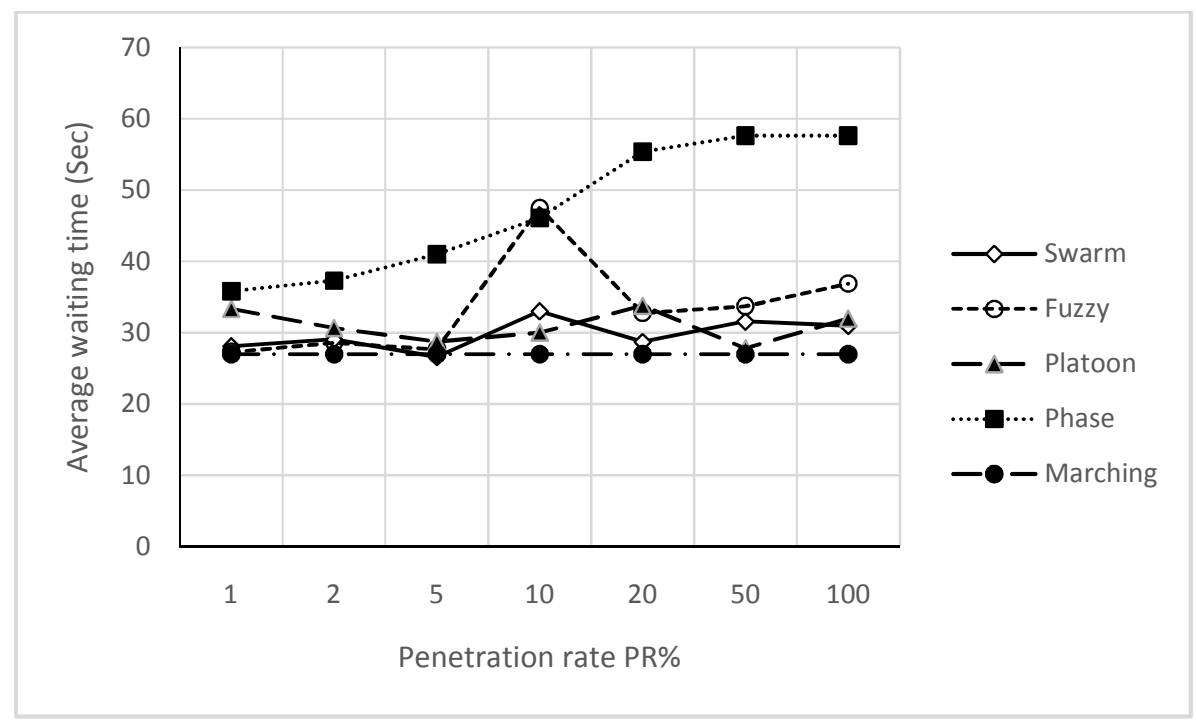

Figure 3. Average waiting time vs. penetration rates

Time loss under different PR, shown in Fig. 4, depicts the time lost due to driving below the ideal speed. Finally, the Fig. 4: Time loss vs. penetration rates duration time under different PR, shown in Fig. 5, depicts the time the vehicle needed to accomplish their route. 
These figures (i.e. Fig. 3-5) show the policies behaviour when simulated each one alone as well as with swarm and fuzzy algorithms in COLOMBO framework with different PR. To evaluate our approach with over mentioned ones, a simple comparison can be made in the following section

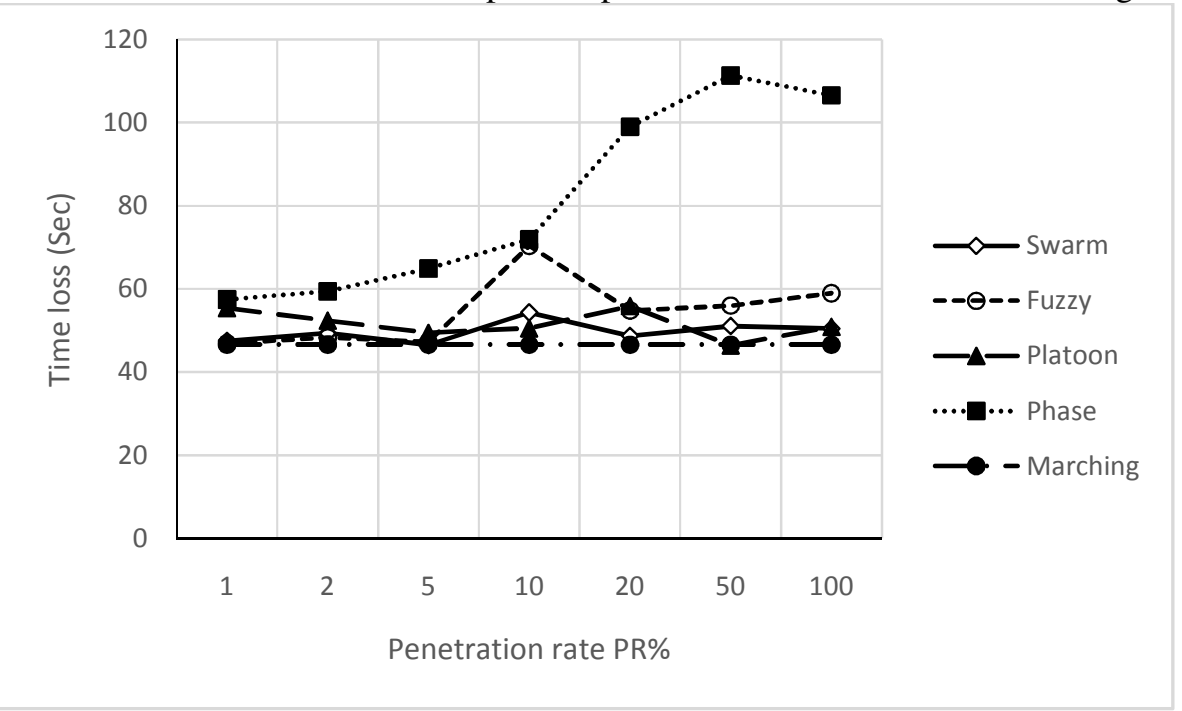

Figure 3. Time loss vs. penetration rates

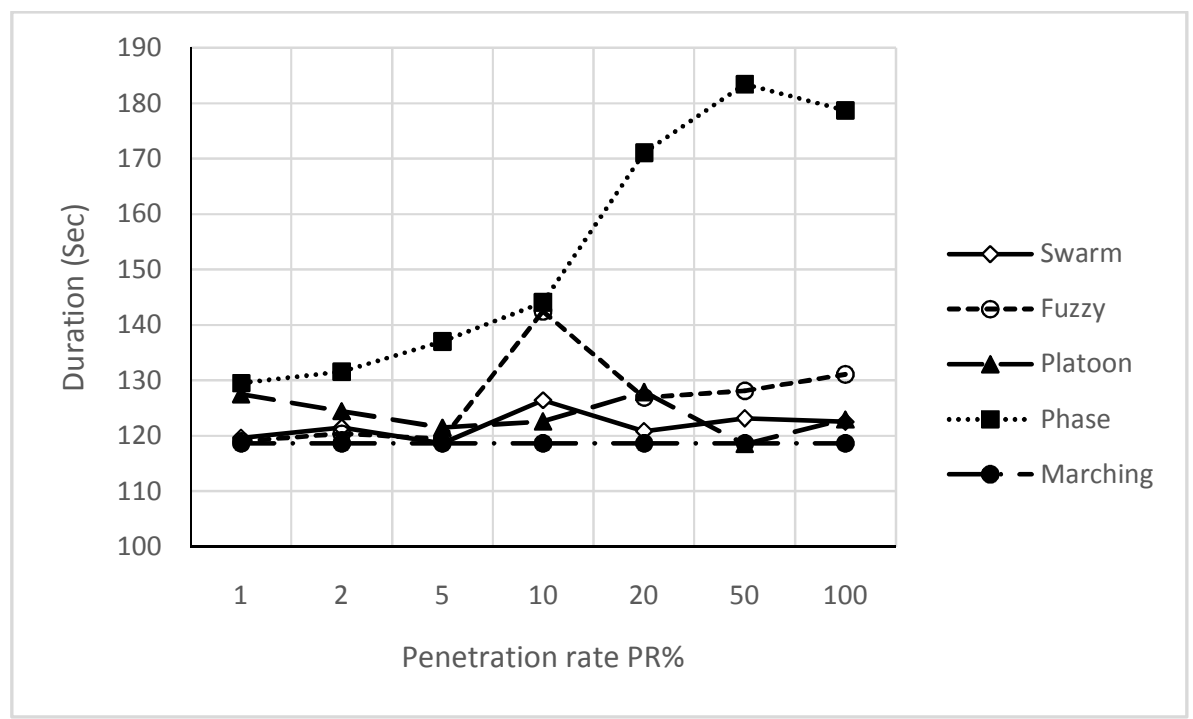

Figure 4.Duration vs. penetration rates

\section{COMPARISON}

In order to evaluate our approach, a simple comparison with COLOMBO approach is done here. Fig. 3-5 shows that marching policy works well for all PR. This policy adopts a static approach (i.e. constant TSC setting) with dynamic phase selection. Since RILSA intersection simulated using only two phases (i.e. no need for dynamic phase selection) and using COLOMBO framework (i.e. with optimized parameters), this may reflect some sort of optimized results with this policy. 
Phase policy fits well for low PR while it does not for high PR. This policy maintains the green light as long as there are no cars on the other directions. The results prove that this behaviour is desirable when there is a dominant traffic flow (i.e. high traffic flow even under low PR) opposed by a irregular one. Platoon policy gets significant results in low-medium PR (i.e. 2-20\%) with minimum values at $50 \%$. This policy creates platoons of vehicles that are free to leave the intersection since nothing blocks them after they pass the central area. Creating platoon required some characteristic, such as platoon size, length and period, to be available to study their behaviour. These characteristics are difficult to study under PPR especially for adaptive problem. The above results clearly indicate that PPR should be handled under specific policies.

Best TSC approach should be able to properly detect each policy situation. Swarm algorithm (more specifically, policy selection procedure of it) select between the above policies with dynamic mechanism. This mechanism depend on parameters optimization that provide smooth transitions between policies. In spite of that, this transition has a clear oscillation effect with $\mathrm{PR}_{\boldsymbol{i}} 10 \%$. In other words, swarm algorithm has an optimization solution with stable transition effect rather than direct policy selection procedure. In the other hand, using simple and direct if then rules (with fuzzy delay estimation)give the same behaviour as for individual policies based on threshold values for policy selection procedure. Both of the above policy selection procedure give comparable results with different PR except at $10 \%$. The results are quite interesting since they show that our proposal is capable to maintain the same performance regardless the PR of equipped vehicles. These comparable results led us to the following section of conclusion.

\section{CONCLUSION}

From the previous comparable results, some conclusions can be stated here. Our TSC solution includes several simplifications compared to COLOMBO's one, taking into consideration PPR based approach. The policy selection procedure in COLOMBO's solution is not relevant for the calculation of TSC setting with low PR because it rely on counting vehicles.

This can be problematic if not all vehicles are sensed like in PPR based approach. That's why different versions of policy selection procedure had been proposed as an optimization problem in COLOMBO's solutions. In the other hand, our solution has the very positive logical behavior of policy selection procedure. The TSC evaluation strongly depend on the policy selection procedure. Using threshold values, as in our solution for policy selection procedure, make it open for further optimization. With these threshold values our solution can get almost the same performance even when one vehicle is sensed under the RSU communication range without relying on counting vehicle. This motivates the solution to be used for PPR based approach. At the same time, focusing on developing policy selection procedure with threshold values based on delay time estimation is not enough. In fact, as shown in Fig. 5, the duration for each vehicle to accomplish their route give an indicator for another parameter should be taken into consideration, evaluation period. Most TSC evaluated for typically 15 minutes as said before. But none of the compared approach for policy selection procedure had been taken this period into consideration. One obvious issue is the lack of data for intervals where no equipped vehicle was sensed. The probability to have no data for an interval depends on the aggregation interval's duration and the PR. For this reason, low PRs show data lacks at times where no equipped vehicle has been within the communication range. As a result, our approach should be further investigate by taking evaluation period into consideration.

\section{REFERENCES}

[1] M. Ferreira, R. Fernandes, H. Conceicao, W. Viriyasitav at, and O.Tonguz. (2010) "Self-organized traffic control". In Proceedings of the 7th ACM international workshop on Vehicular Internetworking. pp. 85-90. ACM,. 
[2] Hugo Conceicao, Michel Ferreira and Peter Steenkiste(2013) "Virtual Traffic Lights in Partial Deployment Scenarios," IEEE Intelligent Vehicles Symposium(IV) June 23-26, Gold Coast, Australia.

[3] Gershenson, C.(2005)"Self-organizing Traffic Lights,” Complex Systems, Vol.16, pp. 2953. 2005.

[4] Priemer, C., Friedrich, B. (2009)"A decentralized adaptive traffic signal control using V2I communication data," 12th International IEEE Conference on Intelligent Transportation Systems, ITSC 09. pp. 16. 2009.doi:10.1109/ITSC.2009.5309870

[5] Datesh, J.; Scherer, W.T.; Smith, B.L. (2011) "Using k-means clustering to improve traffic signal efficacy in an IntelliDriveSM environment," 2011IEEE Forum on Integrated and Sustainable Transportation System(FISTS). pp. 122-127. doi:10.1109/FISTS.2011.5973659

[6] He, Q.; Head, K.L.; Ding, J. (2012)"PAMSCOD: Platoon-based arterial multi-modal signal control with online data," Transportation Research Part C: Emerging Technologies. Vol. 20, pp. 164-184. 2012.doi:10.1016/j.trc.2011.05.007

[7] Lee, J.; Park, B.; Yun, I. (2013)"Cumulative Travel-Time Responsive Real-Time Intersection Control Algorithm in the Connected Vehicle Environment," Journal of Transportation Engineering. Vol. 139, pp. 10201029. 2013.doi:10.1061/(ASCE)TE.1943-5436.0000587

[8] Goodall, N.; Smith, B.; Park, B., (2013)"Traffic Signal Control with Connected Vehicles," Transportation Research Rec. Journal of Transportation Research Board, Vol. 2381, pp. 65-72. doi:10.3141/2381-08.

[9] EU FP7 COLOMBO Project, (2012-2015)[Available Online]: http://www.COLOMBO-fp7.eu/ [Accessed 12 June 2016].

[10] RiLSA1, COLOMBO, (2014). [Available Online]: http://sourceforge.net/projects/sumo/files/trafficdata/scenarios/RiLSA/ [Accessed 12 June2016].

[11] COLOMBO, Deliverable 2.2 (2014),"Policy Definition and dynamic Policy Selection Algorithms". [Available Online]: http://www.COLOMBOfp7.eu/[Accessed 12 June 2016].

\section{AUTHORS}

Muntaser A. Salman has been with College of computer science and information technology at university of Anbar, Anbar, Iraq, since 2003. He received his B.Sc. and M.Sc. degrees in electrical engineering from Basrah University, Basrah, Iraq, in 1997 and2000 respectively.

Currently, he is working toward the Ph.D. degree at Computer Engineering Department, Institute of Engineering and Natural Sciences, Yildirim Beyazit University, Ankara, Turkey. His research interests include computer networks, vehicular networks, computational intelligence algorithms and intelligent transportation systems

Suat Ozdemir has been with the Computer Engineering Department at Gazi University, Ankara, Turkey, since 2007. He received his MSc degree from Syracuse University and PhD degree from Arizona State University in 2001 and 2006, respectively.

Dr.Ozdemir's research are as mainly include wireless and sensor networks, network security, and data mining. He is a member of IEEE and currently serving as editorial

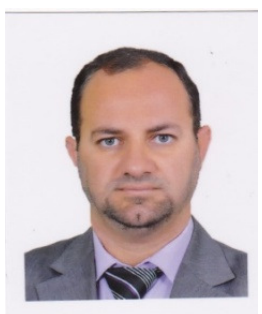
board or TPC member for various leading IEEE and ACM journals and conferences.

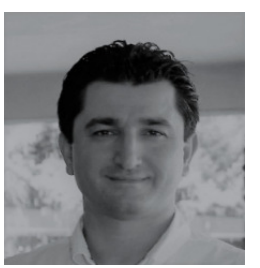


Fatih V. Celebi was born in Kahramanmar as, Turkey, 1963. He received his B.Sc. and M.Sc.degrees in electrical and electronics engineering from Middle East Technical University (METU) and Gaziantep University, Turkey, in 1988 and 1995,respectively.

He earned his Ph.D. degree in Electronics/Computer engineering from Erciyes University in 2002. His research interests include Artificial Intelligence Techniques and Optical Design. He is currently a full professor and Vice president Yildirim Beyazit University, Ankara, Turkey.

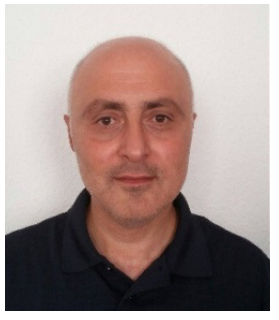

